\title{
Mediating Role of Time Pressure on the Relationship between Organizational Psychology Safety and Motivation of Learning from Failure in Large-Scale Construction Project
}

\author{
Lingna Lin $\mathbb{D D}^{1}{ }^{1}$ Xiaowei Luo, ${ }^{2}$ Sainan Cheng, ${ }^{1}$ Min $X{ }^{1},{ }^{1}$ and Lianbo $\mathrm{Zhu}^{1}$ \\ ${ }^{1}$ School of Civil Engineering, Suzhou University of Science and Technology, 1701 Binhe Road, Gaoxing District, Suzhou 215011, \\ Jiangsu, China \\ ${ }^{2}$ Department of Architecture and Civil Engineering, City University of Hong Kong, Hong Kong, China
}

Correspondence should be addressed to Lingna Lin; linlingna1010@126.com

Received 24 September 2021; Accepted 25 January 2022; Published 10 February 2022

Academic Editor: Luigi Aldieri

Copyright (c) 2022 Lingna Lin et al. This is an open access article distributed under the Creative Commons Attribution License, which permits unrestricted use, distribution, and reproduction in any medium, provided the original work is properly cited.

Large-scale construction projects are characterized by the temporary cooperation of different parties with objectives, making it challenging to learn from failure under great time pressures. Learning from failure in such multiparties projects must overcome the fear of speaking out the "failure," reflected by organizational psychology safety. This study aims to explore the impact of organizational psychology safety on the motivation of learning from failure under the mediating role of time pressure by a crosssectional study. Data was collected by a questionnaire survey of 189 construction practitioners and analyzed by applying regression analysis. Results show that organizational psychology safety was negatively associated with time pressure and positively with the motivation of learning from failure. Time pressure was detrimental to the motivation of learning from failure, and it partially mediated the relationship between organizational psychology safety and motivation of learning from failure. Insights from the analysis could help the managers of organizations or projects to attach importance to the influence and role of learning from failure and could provide them with guides to implement lessons-learned systems.

\section{Introduction}

Large-scale construction projects (LCPs), organizations, and technology platforms for value-added activities [1] reflect high degrees of complexity. In an LCP, participants from different stakeholders (e.g., contractors, subcontractors, owners/investors, designers, and suppliers) get together to conduct project activities and share knowledge for producing, exploiting, and executing solutions to promote innovation and to achieve a common goal. Therefore, the nature of large-scale construction projects is cross-organization structures [2]. The cross-organization and temporal efforts characteristics make it more challenging to learn from failure and use the lessons learned for the other projects [3]. LCPs have failed to communicate the failed-project information effectively and have not yet formed a culture of learning from failure (LF) that links technology and people together. When the stakeholders move to the next project, the same mistakes might be repeated as those they made before.

Considering the efforts required for LF in LCPs, the conflict between project objectives (i.e., cost, quality, and schedule) and learning objective is inevitable. Previous studies $[4,5]$ indicate that time pressure has a potential influence on the motivation of learning from failure (LFM). Time is a double-edged sword for learning from failure behavior in LCPs. On the one hand, the long durations of LCPs provide participants the opportunities to continuously learn from failures [6]. On the other hand, time pressure, combined with resource constraints, affects the project team's LFM [7]. Under high time pressure, the project team members from different organizations have only a limited time to get to know each other as they might not have experience of working together before. They must learn 
collaboratively, quickly, and effectively. The learning behaviors of participants will hence change accordingly under TP [8]. Therefore, it is necessary to consider the relationship between participants' LFM and TP in the context of LCPs.

Under TP, LF plays an extra-task role, and it is not instantaneous or unconscious [9], and psychological and emotional obstacles can accompany failure experiences. These obstacles can be accepted naturally as a result of linked grief, misery, and remorse, but because of facing failure, one's potential misstep can be a dispiriting situation. In addition, the interpretation of failure functions as a pivotal role in learning activities because it acts as a "mental warning" [10] to change participants' mental models [8]. The demonstration has displayed that members generally pay no attention to learning and might be incapable of LF [11]. Such 'mental warning' makes participants grouchy even hostile, impeding their competence to learn from failures [12]. Constrained by psychology and emotion, organization managers seem to pay more attention to project objectives [13], resulting in the negligence or hindrance of LF activities. In particular, LCPOs are usually under high schedule pressure $[14,15]$ when they face the goals of completing project tasks and LF at the same time [16-18].

Different from organizational learning theory, LF is more complicated than general organizational learning due to the sensitivity of failure term. According to Grewatsch and Kleindienst [19], the aforementioned behaviors or actions toward management can be explained by psychological theory. The social psychology research literature provides the basis for filling the existing gap in understanding the relationship between LCPs' failures and learners' motivation in learning. Members of an organization may feel negative or afraid of others' bias against their mistakes when they work with members from other organizations. According to social cognitive theory, participants' response to project failures rests with their explanation of the failures rather than the failure itself [20]. In other words, different participants have different sentiments of the same failure, leading to different decision-making behaviors. Moreover, the notion of organizational psychology safety (OPS) in influencing LF behavior is recognized as highly important to the organization and interorganizations [15], which involve traditional working space [21], manufacturing plants [22], and project teams [23-25]. Field studies have shown that the organizational LF is driven by psychological perceptions and concerns, and the lack of OPS inhibits organization's learning behaviors, such as involvement in an experiment, seeking help, or questioning current team practices $[23,26]$. OPS is regarded as a characteristic of organizational culture [27] and a team characteristic of the shaping behaviors of team leaders [23]. This study believes that the combination of the project-nature element and OPS should be considered in the research of LF in LCPOs. Therefore, this study starts with one of the key characteristics of LCPs (namely, TP) and the feature of extra-role (OPS) to better connect the relationship of interorganizational cooperative learning in LCPs.

Despite the progress, there has been no systematic framework to make the OPS in the LF process clear in LCPOs. Existing studies mainly investigated the positive impact of individual's psychology safety on LF within the organization, instead of the role of OPS of different organizations in an LCPO in the LF process. Specifically, how psychology safety among organizations affects LF has been seldom studied. This study aims to develop the auxiliary mechanisms that construction leaders could adopt to improve their lessons-learned system and LF practices. Quantification of the linkage between OPS and LFM and the mediating role of TP in this relationship could explain interactions and the internal mechanisms of LF. To address this challenge, this study (1) explores the impacts of OPS on LFM; (2) identifies the mediating role of TP on the relationship between OPS and LFM; and (3) develops a framework of the auxiliary mechanisms of LF to improve OPS and decrease TP effectively.

\section{Literature Review}

2.1. Motivation of Learning from Failure. Comparing with intraorganizational learning, cross-organizational LF has the potential to add value for the projects and organizations of LCPOs. Such a learning pattern can make up for the obstacle of learning motivation caused by the limitation of intraorganizational learning. For example, much overlapping knowledge within an organization results in high learning costs and repetitive learning rather than increasing the value added of the projects or organizations [28]. By adopting the best knowledge practices, LCPOs may maintain and improve their competitiveness, productivity, and innovation. These benefits can be translated and applied to the construction environment, such as cost deductions, improvement of quality, productivity, and customer satisfaction. Gegenfurtner [28] analyzed the moderating effects of knowledge type and knowledge evaluation conditions on knowledge sharing motivation and transfer. Recently, research has focused on project failures and the promotion of learning organizations. An increasing number of construction and engineering organizations have the urgent motivation to develop into learning organizations and continue to learn from the outside world's failure experiences.

The etiology theory of organizational climate shows that the existence of various poor climate in an organization has a great relationship with its organizational function [29]. Faced with similar mechanism environments (e.g., knowledge sharing incentive) in an organizational LF, individuals have relatively identical cognition of these mechanisms, such as fear of speaking. According to an explanation of climate etiology provided by Schneider and Reichers [29], the organizational settings influence people's attitudes, concepts, and opinions on organizational events, thus affecting the learning motivation.

2.2. Organizational Psychology Safety. Psychology safety was originally an element used to study individual psychological characteristics [23]. Zhang et al. [15] indicated that psychology safety is better served as a teamlevel climate, which can promote teams' learning 
behaviors, and the main reason is that it lessens attention to which some members may react passively to the others' learning behaviors. Thus, based on team context, research has identified psychological safety as an important factor in understanding how teams (e.g., entrepreneurial team, medical team) work together to achieve shared outcomes [30]. It is considered as an effective factor decreasing the level of blame and hostility about project failures for members [31], which has been empirically supported by other studies [15, 30-33]. Carmeli and Gittell [34] adjusted Edmondson's sevenitem scale of team psychological safety, and the empirical research on an organizational level is carried out. Here, organizational psychology safety (OPS) refers to a shared belief that organizations are safe when they take cooperative risks [34].

The psychological theory of dealing with failures is a useful theoretical basis for the model of participants' moving up from project failures [13]. OPS is something beyond trust [23], and it may relieve the unwillingness of participants to deliver themselves by lessening the fear of disgrace or unpopularity [7]. Recent research showed that OPS is significant to LF among participants in the context of Architecture, Engineering, and Construction (AEC) fields [25]; whether it is from the perspectives of organizational structure, culture, social interaction, or psychological perception, creating an OPS climate in those organizations is conducive to the improvement of safety culture and organizational learning atmosphere [31].

2.3. Time Pressure. Time pressure and the temporary nature of the project mean that the end of the project is usually the end of the colearning [35]. LF often proceeds after the project events, which shows a time lag. Keegan and Turner [36] found that TP is one of the key factors of the learning of project-based organizations through a survey of 19 European companies engaged in different industries. Based on the principal-agent theory, Lin and Wang [37] conducted a simulation analysis of multiagent project team knowledge sharing and pointed out that the knowledge sharing effort level of project team participants decreased with the passing of time. In other words, limited time leaves organizations with no systematic way to decide what actions to take or not to take. Particularly in LCPs, most participants have technical backgrounds, which leads to the fact that accident analysis often focuses on technical problems and actions to improve the technology, and human and organizational problems are rarely solved [38]. Therefore, a lack of time or budget is often the result of management decisions [39]. The time variable can be used as an intra-role characteristic of a project [14], or as an antecedent of extra-role (i.e., failure learning) characteristic of a project [4]. Therefore, identifying and analyzing the impact mechanism of time variable on cross-organizational LF are conducive to the realization of project value added under the premise of achieving project objectives.

\section{Hypothesis Development}

3.1. Organizational Psychology Safety in Large-Scale Construction Project Organizations. OPS is context-specific and is most conspicuous in contexts where organizations' involvement in specific behaviors puts their habits or interests in jeopardy (namely, latent passive outcomes could result from the behaviors). For example, when OPS is high, participants will feel confident that their behaviors are safe and will not lead to adverse outcomes, which in turn allows them to keep their behaviors, such as constructive voice [40]. Tucker et al. [41] studied the hospital intensive-care units and found that OPS is positively related to learning behaviors, which eventually forms successful implementation of new practices. Liu et al. [42] found that OPS can improve teams and individuals' learning across multiple organizations.

Organizations with high OPS create a situation in which they actively face challenges that may go against their expectations or hopes without triggering defensive responses [30] and organizational bullying [43]. For example, Shen et al. [31] stressed the importance of psychological safety climate on construction sites and provided three paths for the improvement of psychological safety. Many participants in LCPs, despite their strong self-ability and growing experiences, suffer from the poorly sustained relationship and inclusiveness for mistakes that shake their final success [24]. While previous studies have surveyed psychological safety among individuals [22], in and among teams [23,31,34], in and among organizations $[25,41]$, this study concentrates on multiorganizations' psychological safety in the LCPs fields in which they participate.

In the process of cross-organizational cooperation, participants' self-expressive behaviors in the form of failurebased learning might put themselves at risk because the affiliated organization can easily identify the root of mistakes, and the responsible person, or even the partners, may question their competence. In such a case, OPS may also be a prominent factor in promoting LF. When psychological safety is perceived, the participants are more likely to express their opinions, present their specialized knowledge and skills, and learn from others in the project team. In other words, they believe that their learning behavior will not lead to adverse outcomes, such as disgrace, blame, or hostility, even if their LFM is not for the whole team. Therefore, the hypothesis is proposed as follows:

H1. LFM in LCPOs is positively correlated with the perceived degree of OPS.

\subsection{Mediating Effect of TP on the Relationship between OPS} and LFM. TP has two sides to organizational learning. According to Chuderski [4] and Thomas et al. [44], TP is one of the key factors that hinder teams from learning. The strategies that members use for learning are influenced by TP [4]. Because of project TP, LCPOs tend to get used to the way of centralized learning and defer learning to the future. For instance, members will spend more time in processing the manageable events, whereas more unmanageable 
fragments will be ignored. It may lead to poor learning effects [45]. In particular, such destructive effects happen when all events and fragments appear simultaneously. In addition, participants will turn to implicit learning when they are affected by TP [8]. For example, participants apply simplified, emotion-driven strategies, which generally are less resultful than the matured and specific ones. However, TP does not always affect learning negatively. According to Schmitt et al. [46], proper TP can promote learning performance, particularly when switching among tasks is required. Despite the diversified outcomes of previous studies, TP can be a challenge or an obstruction to LF.

Existing research analyzed the impact of TP on general learning, such as norms, and successful experiences [5]. According to Silla and Gamero [26], high TP worsens the learning effects when the contents of learning are complicated, and the participants have less cognitive capital. Especially in the LF context, LCPOs have more intense perceptions of schedule. In other words, such discrepant results can be interpreted by the fact that studies have primarily emphasized the direct effect of TP on learning performance, overlooking the motivational and perceptive processes [47] to which TP is connected and that underlie learning performance. From the organizational behavior perspective, LFM is the result of the interaction between the intrinsic factors (i.e., perception, emotion) and external environments (resource constraints, i.e., time, cost). One factor often mentioned in the research is project TP that participants undergo during the implementation process of the projects [14]. OPS is an important antecedent of LFM, and project TP functions as a key resource constraint for project participants [48]. TP is a key antecedent of LFM, and therefore it not only has a direct impact on LFM but also functions as a mediating role by facilitating the perception of OPS.

Regarding LF, OPS is a crucial perceptive belief among participants. LF is influenced by various factors, which can be classified into two primary processes: the motivational process and perceptive process [47]. In the motivational process, members are most active because their behaviors are motivated mainly by their own interests and challenges of the learning. In the perceptive process, members are most initiative because they are capable of acquiring, assessing, and applying the required knowledge. Given the effect of cognitive TP on FL and the importance of perceptive psychology, the hypotheses that TP can hinder LFM in projects and mediate the relationship between OPS and LFM are proposed as follows:

$\mathrm{H} 2 . \mathrm{TP}$ in LCPOs is negatively correlated with LFM.

H3. TP has a negative mediating effect on the relationship between OPS and LFM. That is, the higher the perceived TP, the smaller the influence of OPS on LFM.

3.3. Theoretical Model. As discussed above, this study aims to identify the effect mechanism of OPS and TP on LFM in LCPOs. The proposed theoretical model is summarized in Figure 1.

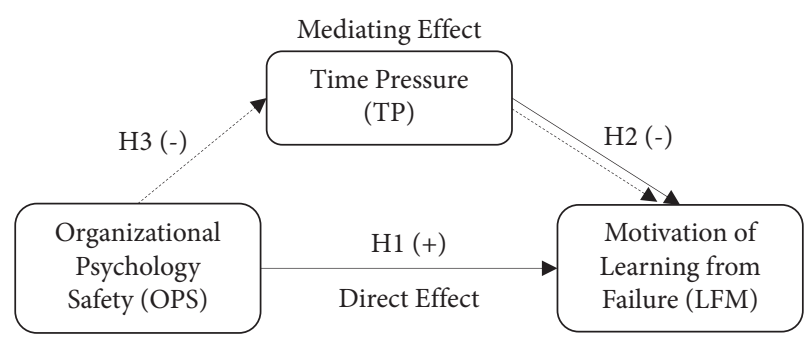

Figure 1: Conceptual Framework. Note: The mediating effect of time pressure is indicated by dashed lines $(\mathrm{H} 3)$.

\section{Research Methodology}

4.1. Questionnaire Development. Pilot research was conducted to adjust the existing measurement items before developing formal questionnaires. The preliminary questionnaires were assessed by five scholars, nine project managers, and two enterprise leaders in the construction field. The experts in this preliminary study ranged in age from 38 to 50 (average $=43.3$ ) and have more than ten-year experience in research or practice in the field of construction project management. Some items were revised or deleted after discussion with these experts. For example, psychological safety at the team level was measured by Edmondson [23]. To maintain the theoretical conceptualization of the assessed construct [34], the authors replaced the word "team" originally used by Edmondson [23], with the word "organization." The measurement scale of LFM is based on Tucker and Edmondson's [49] failure-based learning behaviors and Kyndt et al.'s [50] learning motivation, and the preliminary items of LFM were developed and constructed with a four-item scale. For the original items translated into Chinese, back-translation was thus applied to ensure conceptual equivalence [51]. Eventually, the final questionnaire consists of two parts: basic information and questions.

4.2. Construct Measures. Measures of LFM, OPS, and TP were based on previous studies [23, 25, 34, 46, 49, 50] and adapted to the contexts and characteristics of this particular study. The following items were included.

4.2.1. LFM. "The purpose of talking about the failure-related experience is to avoid mistakes in the follow-up tasks rather than to blame others." "Our organization encourages us to talk with persons in our surroundings about the failurerelated experience in order to look for solutions." "Our organization encourages us to talk with persons in our surroundings about the failure-related experience not only to acquire solutions of the task at hand, but also to find the root cause of the problems." "In our organization, members are encouraged to ask questions such as 'is there a better way to produce the product or provide the service."”

4.2.2. OPS. "Members of this organization can bring up problems and tough issues." "It is safe to take a risk in this organization." "No one in this organization would 
deliberately act in a way that would undermine my efforts." "Working with members of this organization, my unique skills and talents are valued and utilized."

4.2.3. TP. "In my project-based organization we have not much time to learn from failure at work." "I have to neglect some tasks because I have too much to do." "Different groups at work demand things from me that are hard to combine." "I am unable to take sufficient breaks." All items were rated on a 5-point Likert scale ranging from $1=$ "strongly disagree" to $5=$ "completely agree."

4.3. Data Collection. Two hundred fifty questionnaires were delivered to the owners, contractors, consultants, suppliers, and designers of LCPs in China through two distributed ways: on-site of different cities and by e-mail. One hundred eighty-nine valid questionnaires were received, making the response rate $75.6 \%$. Table 1 summarized the distribution of respondents' profile.

\section{Data Analysis and Results}

5.1. Data Analysis. Cronbach's $\alpha$ was calculated using SPSS 17.0 to evaluate the internal consistency and reliability of the scales. Employing two-step procedures from [52], a measurement model was explored to assess the model's adequacy via performing a confirmatory factor analysis (CFA), and a structural equation model was established to test the research hypotheses. Twelve items constituted the measurement model, and the three first-order constructs were estimated. The measurement model is validated by reliability and validity. We also tested the mediating effect of TP on the relationship between OPS and LFM (H3).

5.1.1. Measurement Reliability and Construct Validity. Factor analysis of the 12 items resulted in a 3-factor solution that accounted for $68.98 \%$ of the total variance. All items had sufficient loadings (above 0.40) on Haman's single-factor test. Cronbach's $\alpha$ for the three constructs were 0.761 (LFM), 0.903 (OPS), and 0.842 (TP). Table 2 indicated strong reliability as the composite reliabilities (CR) were all greater than 0.7 benchmarks. The average variance extracted (AVE) values exceeded the 0.5 cutoff. Therefore, these measures revealed sufficient convergent validity. All the three measurements' Kaiser-Meyer-Olkin (KMO) statistic was 0.8, indicating that the strong correlation of variables was suitable for factor analysis.

5.1.2. Structural Model. In the following model, the authors applied the path model to examine the proposed model (Figure 2). The model fit indices $\left(\chi^{2}=84.681\right.$, $p=0.001, \mathrm{df}=48, \mathrm{RMSEA}=0.064, \mathrm{IFI}=0.969, \mathrm{CFI}=0.968$, $\mathrm{TLI}=0.957$, and NFI $=0.931)$ manifested that the fitting between the model and data was good.

The Pearson correlation was carried out to confirm the relationships among different variables (Table 3 ). The coefficients about means, standard deviations, and correlations
TABLE 1: Distribution of the respondents' answers.

\begin{tabular}{lcc}
\hline Variables & Frequency & Percentage \\
\hline Gender & 121 & \\
Male & 68 & 64.02 \\
Female & & 35.98 \\
Age & 26 & 13.76 \\
$<30$ & 154 & 81.48 \\
$30-50$ & 9 & 4.76 \\
$>50$ & & \\
Education level & 6 & 3.17 \\
High school diploma & 91 & 48.15 \\
Bachelor degree & 92 & 48.68 \\
Graduate degree & & \\
Tenure in the present project team & 58 & 30.69 \\
$<1$ year & 108 & 57.14 \\
1-3 years & 23 & 12.17 \\
$>3$ years & & \\
Position & 56 & 29.63 \\
Operator & 81 & 42.86 \\
Supervisor & 35 & 18.52 \\
Assistant manager in section/project & 17 & 8.99 \\
Senior manager & & \\
Number of projects involved in for the recent five years \\
0-1 & 5 & 2.65 \\
2-3 & 48 & 25.4 \\
$4-5$ & 86 & 45.5 \\
More than 5 & 50 & 26.46 \\
\hline
\end{tabular}

are shown in Table 3. Consistent with previous researches (e.g., $[23,34]$ ), OPS is positively related to LFM, $\beta=0.415$, $p<0.05$. OPS is significantly negatively related to TP, $\beta=-0.258, p<0.01$. TP is negatively correlated with LFM, $\beta=-0.432, p<0.01$.

5.1.3. Direct Effect. According to Baron and Kenny [53], the mediated regression analysis can be used for examining the hypotheses. The effect of multicollinearity among variables was eliminated by centering the independent variables before interactive items were formed. Three separate regression equations were examined. First, the authors entered LFM as the dependent variable and the controls (gender, age, education level, tenure in the present project team, position, and number of projects involved in for the recent five years) as independent variables. The results are exhibited in Table 4 . The direct effects contain three models with LFM as the dependent variable. Model 1 only contains control variables. Model 2 combines both the control variables and OPS as independent variables. OPS was a positively direct effect on LFM $(\beta=0.420, p<0.001)$. Model 3 shows that TP was a negatively direct effect on LFM $(\beta=-0.443, p<0.001)$. Therefore, $\mathrm{H} 1$ and $\mathrm{H} 2$ were supported.

5.1.4. Mediating Effect. As shown in Table 4, mediated analyses were conducted to examine the mediating effect of TP on the relationship between OPS and LFM. Firstly, the positive correlation between OPS and LFM was tested $(\beta=0.420, p<0.001)$ without entering the mediator. The level of relationship between OPS and LFM $(\beta=0.420$, 
TABLE 2: Factors' loading matrix, CR and AVE values $(N=189)$.

\begin{tabular}{|c|c|c|c|}
\hline \multirow{2}{*}{ Items } & \multicolumn{3}{|c|}{ Components } \\
\hline & OPS & $\mathrm{TP}$ & LFM \\
\hline OPS3 ("no one in this organization would deliberately act in a way that would undermine my efforts") & 0.894 & -0.148 & 0.115 \\
\hline OPS4 ("working with members of this organization, my unique skills and talents are valued and utilized") & 0.860 & -0.171 & 0.071 \\
\hline OPS2 ("it is safe to take a risk in this organization") & 0.860 & -0.041 & 0.223 \\
\hline OPS1 ("members of this organization can bring up problems and tough issues") & 0.824 & -0.024 & 0.195 \\
\hline TP1 ("in my project-based organization, we have not much time to learn from failure at work") & -0.103 & 0.831 & -0.217 \\
\hline TP2 ("I have to neglect some tasks because I have too much to do") & -0.052 & 0.804 & -0.123 \\
\hline TP3 ("different groups at work demand things from me that are hard to combine") & -0.184 & 0.783 & -0.215 \\
\hline TP4 ("I am unable to take sufficient breaks") & -0.033 & 0.781 & -0.045 \\
\hline $\begin{array}{l}\text { LFM2 ("our organization encourages us to talk with persons in our surroundings about the failure-related } \\
\text { experience in order to look for solutions") }\end{array}$ & 0.183 & -0.117 & 0.835 \\
\hline $\begin{array}{l}\text { LFM1 ("the purpose of talking about the failure-related experience is to avoid mistakes in the follow-up tasks } \\
\text { rather than to blame others") }\end{array}$ & 0.007 & -0.045 & 0.801 \\
\hline $\begin{array}{l}\text { LFM3 ("our organization encourages us to talk with persons in our surroundings about the failure-related } \\
\text { experience not only to acquire solutions of the task at hand, but also to find the root cause of the problems") }\end{array}$ & 0.292 & -0.324 & 0.659 \\
\hline $\begin{array}{l}\text { LFM4 ("in our organization, members are encouraged to ask questions such as is there a better way to produce } \\
\text { the product or provide the service") }\end{array}$ & 0.258 & -0.303 & 0.517 \\
\hline CR (composite reliability) & 0.919 & 0.877 & 0.801 \\
\hline AVE (average variance extracted values) & 0.74 & 0.64 & 0.51 \\
\hline
\end{tabular}

Note: PS1-4: items of OPS (organizational psychology safety); TP1-4: items of TP (time pressure), LFM1-4: items of LFM (motivation of learning from failure); CR: composite reliability; AVE: average variance extracted values.

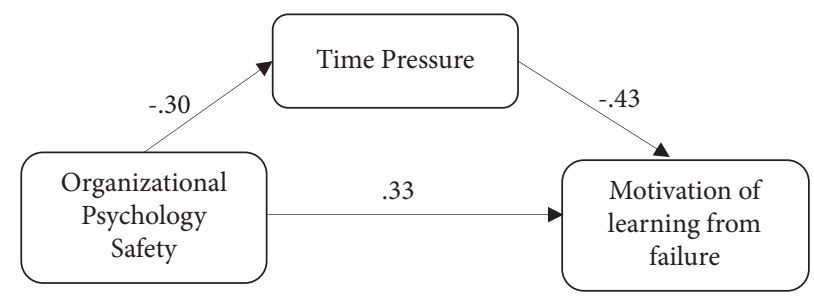

Figure 2: Path model for relations among the latent variables.

TABLE 3: Descriptive statistics and correlation coefficient of variables.

\begin{tabular}{|c|c|c|c|c|c|c|c|c|c|c|c|}
\hline & Mean value & Standard deviation & $\mathrm{G}$ & $\mathrm{T}$ & EL & $p$ & $\mathrm{~A}$ & $\mathrm{~N}$ & TP & LFM & OPS \\
\hline $\mathrm{G}$ & 1.21 & 0.406 & 1 & & & & & & & & \\
\hline $\mathrm{T}$ & 2.62 & 0.813 & -0.118 & 1 & & & & & & & \\
\hline EL & 2.4 & 0.501 & -0.039 & 0.015 & 1 & & & & & & \\
\hline $\mathrm{P}$ & 1.71 & 0.953 & 0.016 & $0.698^{* *}$ & $0.172^{*}$ & 1 & & & & & \\
\hline A & 1.84 & 1.115 & -0.042 & $0.583^{* *}$ & 0.088 & $0.732^{* *}$ & 1 & & & & \\
\hline $\mathrm{N}$ & 2.94 & 0.969 & -0.01 & $0.674^{* *}$ & 0.103 & $0.518^{* *}$ & $0.410^{* *}$ & 1 & & & \\
\hline $\mathrm{TP}$ & 3.725 & 0.855 & -0.1 & 0.046 & -0.094 & -0.064 & -0.094 & 0.058 & 1 & & \\
\hline LFM & 2.318 & 0.637 & -0.008 & 0.031 & 0.07 & 0.014 & 0.025 & -0.035 & $-0.432^{* *}$ & 1 & \\
\hline OPS & 2.922 & 0.945 & -0.02 & -0.03 & 0.074 & 0.036 & 0.087 & -0.017 & $-0.258^{* *}$ & $0.415^{* *}$ & 1 \\
\hline
\end{tabular}

Note: $G=$ gender; $T=$ tenure in the present project team; $\mathrm{EL}=$ education level; $P=$ position; $N=$ number of projects involved in for the past 5 years; $\mathrm{TP}=$ time pressure; $\mathrm{LFM}=$ motivation of learning from failure; OPS $=$ organizational psychological safety. ${ }^{* *} p<0.01 ;{ }^{*} p<0.05$.

$p<0.001)$ was decreased $(\beta=0.333, p<0.001)$ after adding TP. Meanwhile, TP was still negatively correlated with LFM $(\beta=-0.361, p<0.001)$, which means TP has a partial mediating effect. Therefore, H3 was supported.

5.2. Results. First, from the empirical analysis, LFM of LCPOs' participants is greatly influenced by OPS and TP. The positive effect of OPS on LFM $(\beta=0.420, p<0.001)$ helps participants discuss openly and freely and makes them not afraid of being punished and blamed by managers and therefore willing to embrace failures. This result was apparent during the interview; some respondents believed that "when others indicate the conflicts, they do not suppress us. Instead, they are willing to communicate with us to improve the learning." Such perceptions show that participants' high degree of psychological safety plays an important role in LFM. Although it is hard to improve individuals' psychological safety directly, psychological safety may provide a basis for further development of learning from failure and LFM. 
TABLe 4: Parameter estimates of the performed multilevel linear models.

\begin{tabular}{|c|c|c|c|c|}
\hline \multirow{3}{*}{ Variables } & \multicolumn{3}{|c|}{ Direct effect } & \multirow[t]{2}{*}{ Mediating effect } \\
\hline & \multicolumn{3}{|c|}{ LFM as the dependent variable } & \\
\hline & Model 1 & Model 2 & Model 3 & Model 4 \\
\hline \multicolumn{5}{|l|}{ Control variables } \\
\hline $\mathrm{G}$ (gender) & 0.011 & 0.022 & -0.031 & -0.014 \\
\hline $\mathrm{T}$ (tenure in the present project team) & 0.128 & 0.178 & 0.173 & 0.204 \\
\hline A (age) & 0.027 & -0.037 & -0.034 & -0.074 \\
\hline EL (education level) & 0.086 & 0.060 & 0.048 & 0.035 \\
\hline $\mathrm{P}$ (position) & -0.051 & -0.050 & -0.078 & -0.073 \\
\hline $\mathrm{N}$ (number of projects involved in for the past 5 years) & -0.115 & -0.112 & -0.077 & -0.082 \\
\hline \multicolumn{5}{|l|}{ Independent variables } \\
\hline OPS & & $0.420^{* * *}$ & & $0.333^{* * *}$ \\
\hline $\mathrm{TP}$ & & & $-0.443^{* * *}$ & \\
\hline \multicolumn{5}{|l|}{ Mediating variables } \\
\hline $\mathrm{TP}$ & & & & $-0.361^{* * *}$ \\
\hline$R^{2}$ & 0.014 & 0.186 & 0.201 & 0.303 \\
\hline$\Delta \mathrm{R}^{2}$ & - & $0.172^{* * *}$ & $0.187^{* * *}$ & $0.117^{* * *}$ \\
\hline $\mathrm{F}$ & 0.426 & $5.912^{* * *}$ & $6.507^{* * *}$ & $9.79^{* * *}$ \\
\hline$\Delta \mathrm{F}$ & - & $38.305^{* * *}$ & $42.413^{* * *}$ & $30.248^{* * *}$ \\
\hline
\end{tabular}

Second, OPS was negatively correlated with TP $(\beta=-0.258, p<0.01)$. The result has not been previously verified in LCPOs, although theories have proposed that organizational context is acted as a relevant antecedent of task requirements [3]. LF, on the one hand, involves the roles compatibility of intra- and extra-task. On the other hand, it involves the balance of psychological perception. The higher the PS is, the more flexible the participants are in scheduling tasks in and out of their roles [36].

Third, TP has a negatively direct effect on LFM $(\beta=-0.443, p<0.001)$. Involvement in LF is difficult for participants in LCPs with high TP [54]. LCPs' attributes often prevent LF from occurring because participants focus on time and product, service, and delivery rather than LF activities. TP hence means that the end of a project is usually the end of learning together [35].

TP plays a mediated role in the relationship between OPS and LFM. The findings of this study showed that TP was magnified for some projects if the time was not properly allocated. For example, some respondents agreed that "lots of TPs are concentrated in a certain stage in Chinese LCPs, and most of these pressures come from the owner's unreasonable demands, such as 'the designer is asked to deliver the design change scheme within one day," "the project management company is required to complete the feasibility investigation program within three days, etc." Therefore, high TP will reduce the OPS of LFM. This ultimately brought a reduction in learning behaviors, leading to the essential reasons and experiences of project failure to be ignored.

\section{Discussion}

At present, cost overrun, safety accidents, quality issues, and partners' conflict of LCPs have not been improved with the progress of technical conditions $[3,55]$, which indicates that the failed experiences have not been well learned and accumulated [56]. The purpose of interorganizational cooperation is to realize the added value. Through technical means such as lessons-learned or knowledge management systems and managerial and organizational means such as communities of practice and PMO [35, 57], construction enterprises are encouraged to learn from others, establish sharing models, and create incentive mechanisms, to promote the LF behaviors. However, due to the sensitivity of failures and the sociality of LCPs [12], some construction enterprises tend to hide past failures, and they are unwilling to recall those failures, leading to the passivity of LF. Moreover, even with the emphasis on LF, most organizations tend to focus on major accidents, which results in LF behavior being limited to fewer major cases. Members in LCPs teams often have little time for meetings to evaluate lessons-learned [36]. In general, members are assigned to the next project immediately after the project completion, making them lack time studying experiential learning or postevaluation of behaviors. According to Keegan and Turner [36], none of the respondents expressed satisfaction with the process, and most respondents believed that TP was one of the key obstacles to LF, reducing the effectiveness of learning from these experiences. TP and the temporary nature of the project mean that the end of the project is usually the end of colearning $[35,58]$. Therefore, both OPS and TP play a vital role in the study of LF in LCPs.

TP causes LCPOs to lose enthusiasm for learning (H2) because TP destroys the shared belief that will be formed and developed by participants during the cooperation process. The high degree of TP restricts learning willing by preventing participants from immersing in dialogue and communication and by inducing them to step back on failure blame and hostility rather than learning failures and interpreting them. In other words, TP has a spillover effect on PS [59]. Thus, unmotivated organizations rely only on existing knowledge to execute activities and avoid providing 
innovative solutions to the newly appearing problems, especially when these problems are primarily the responsibility of the others. Tasks and activities of a construction project are given a certain time frame. However, the cooperation among LCPOs often appears to be unstructured and aimless at the early stages [35]. Participants will work hard and focus on the project itself rather than learning when they perceive the TP because of the imminent deadline [36]. The learning activities end when the project is finished. The absence of FL during the project implementation phase makes it difficult for organizations to recreate knowledge in the following daily activities. Therefore, it is precise because of TP that LF is more important and worthy of attention.

The positive effect of OPS on LFM was partially mediated by TP (H3). This indicates that TP is of great importance for organizations to learn from failure. Meanwhile, it also reflects the weakening effect of OPS of LF caused by TP. Put differently, motivation, behaviors, and performance for $\mathrm{FL}$ can be effectively promoted when the LCPOs provide some measures to reduce TP.

The organization managers should ruminate participants who pay attention to the negative perceptions experienced from project failure and how these perceptions can adversely influence the next projects, rather than concentrating on the causes of failures [60]. For example, interactive perspectives such as customer safety participation [61] and involvement in the experiment [62] can be implemented across LCPOs to identify and reduce participants' negative perceptions of failures.

\section{Conclusion}

LF outside organization has always been a difficult issue in LCPOs. This empirical study showed the significance of OPS for understanding LF in LCPs and examined the mediating role of TP in the relationship between OPS and LFM. The findings contributed to the body of knowledge on harmonization between project tasks and learning from failure by exploring how OPS influences LFM, and the mediating role of TP on the relationship between OPS and LFM.

This research also provides several implications for project management practice. First, the significance of OPS in driving LF in LCPOs has been proved. To improve OPS, a possible way is to support LCPOs and create a climate which forms the perception that it is safe to talk about failures and learn from failures in the project teams. Throughout the platform, such as lessons-learned or knowledge management systems, various managers and leaders can express voice behavior and support their dialogue and encourage others to engage in practice. Second, we concluded the partial mediating effect of TP. It is an important finding since a few previous studies have linked the characteristics of LCPs to LF, which highlights the importance of intra-role task allocation in the study of LF behavior with considering TP. Third, LFM on project failures is a driving force to guide participants to keep involving in continuous learning and is a valid forecaster of actual behaviors.

Although this study obtains important research findings, some limitations still exist. First, this is a cross-sectional analysis such that causal relationships cannot be presumed. For instance, one may believe that OPS and TP could be reciprocal, while previous studies support the impact of OPS on knowledge sharing and exchange. Second, the measurement of the OPS of failure-based learning is a general duty concept instead of an industry-specific one and is also crude. Finally, the number of collected samples is relatively small. Project failure is a sensitive word in Chinese construction projects, and some projects may only have one respondent, which cannot fully reflect the nature of failurebased learning. Compared with the success, the lack of samples of failures is a trend. In addition, this study left some unsolved questions. For instance, the dynamic evolution of LF behaviors is still unaccounted. With respect to future research, the longitudinal studies that present the interaction between the different factors of failure-based learning would be effective. Further, the evolution of contextual factors and their influence on failure-based learning would be discussed.

\section{Data Availability}

The data used to support the findings of this research are available from the corresponding author upon request.

\section{Conflicts of Interest}

The authors declare that there are no conflicts of interest.

\section{Acknowledgments}

The authors acknowledge the financial support from the Social Science Foundation of Jiangsu Province of China (18GLC003), PostGraduate Research and Practice Innovation Program of Jiangsu Province of China (Grant no. SJCX21_1412).

\section{References}

[1] J. Lehtinen, A. Peltokorpi, and K. Artto, "Megaprojects as organizational platforms and technology platforms for value creation," International Journal of Project Management, vol. 37, no. 1, pp. 43-58, 2019.

[2] E. Shiu, Z. Jiang, and G. Zaefarian, "Antecedents of behavioural commitment in inter-organizational relationships: A field study of the UK construction industry," Construction Management and Economics, vol. 32, no. 9, pp. 888-903, 2014.

[3] H. R. Baker, S. D. Smith, G. Masterton, and B. Hewlett, "Failures in construction: Learning from everyday forensic engineering," Forensic Engineering, pp. 648-658, 2018.

[4] A. Chuderski, "Time pressure prevents relational learning," Learning and Individual Differences, vol. 49, pp. 361-365, 2016.

[5] C.-Y. Tsai, Y.-H. Chang, and C.-L. Lo, "Learning under time pressure: Learners who think positively achieve superior learning outcomes from creative teaching methods using picture books," Thinking Skills and Creativity, vol. 27, pp. 55-63, 2018.

[6] C. Fiori and M. Kovaka, "Defining megaprojects: Learning from construction at the edge of experience," Construction Research Congress, vol. 183, pp. 1-10, 2005.

[7] M. K. Nasir and B. H. W. Hadikusumo, "System dynamics model of contractual relationships between owner and 
contractor in construction projects," Journal of Management in Engineering, vol. 35, no. 1, Article ID 04018052, 2019.

[8] E. Raes, A. Boon, E. Kyndt, and F. Dochy, "Exploring the occurrence of team learning behaviours in project teams over time," Research Papers in Education, vol. 32, no. 3, pp. 376-401, 2017.

[9] A. Wilkinson and K. Mellahi, "Organizational failure: Introduction to the special issue," Long Range Planning, vol. 38, no. 3, pp. 233-238, 2005.

[10] M. Gartmeier, J. Bauer, H. Gruber, and H. Heid, "Negative knowledge: Understanding professional learning and expertise," Vocations and Learning, vol. 1, no. 2, pp. 87-103, 2008.

[11] D. J. Wei, M. L. Hui, and C. Kang, "The learning status survey of our country construction enterprise employees," Building Economics, vol. 2, no. 2, pp. 91-96, 2013, (in Chinese).

[12] D. A. Shepherd, H. Patzelt, and M. Wolfe, "Moving forward from project failure: Negative emotions, affective commitment, and learning from the experience," Academy of Management Journal, vol. 54, no. 6, pp. 1229-1259, 2011.

[13] H. L. Chen, "Performance measurement and the prediction of capital project failure," International Journal of Project Management, vol. 33, no. 6, pp. 1393-1404, 2015.

[14] A. Kach, A. Azadegan, and K. J. Dooley, "Analyzing the successful development of a high-novelty innovation project under a time-pressured schedule," R\&D Management, vol. 42, no. 5, pp. 377-400, 2012.

[15] Y. Zhang, Y. Fang, K.-K. Wei, and H. Chen, "Exploring the role of psychological safety in promoting the intention to continue sharing knowledge in virtual communities," International Journal of Information Management, vol. 30, no. 5, pp. 425-436, 2010.

[16] P. Carrillo, K. Ruikar, and P. Fuller, "When will we learn? Improving lessons learned practice in construction," International Journal of Project Management, vol. 31, no. 4, pp. 567-578, 2013.

[17] Z. Ren, G. Q. Shen, and X. L. Xue, "Failure caused by inappropriate construction methods: An expensive lesson," Journal of Management in Engineering, vol. 29, no. 1, pp. 25-34, 2013.

[18] D. Sage, A. Dainty, and N. Brookes, "A critical argument in favor of theoretical pluralism: Project failure and the many and varied limitations of project management," International Journal of Project Management, vol. 32, no. 4, pp. 544-555, 2014.

[19] S. Grewatsch and I. Kleindienst, "How organizational cognitive frames affect organizational capabilities: The context of corporate sustainability," Long Range Planning, vol. 51, no. 4, pp. 607-624, 2018.

[20] P. M. Madsen and V. Desai, "No firm is an island: The role of population-level actors in organizational learning from failure," Organization Science, vol. 29, pp. 1-15, 2018.

[21] S. Duffield and S. J. Whitty, "Developing a systemic lessons learned knowledge model for organisational learning through projects," International Journal of Project Management, vol. 33, no. 2, pp. 311-324, 2015.

[22] E. Siemsen, A. V. Roth, S. Balasubramanian, and G. Anand, "The influence of psychological safety and confidence in knowledge on employee knowledge sharing," Manufacturing \& Service Operations Management, vol. 11, no. 3, pp. 429-447, 2009.

[23] A. Edmondson, "Psychological safety and learning behavior in work teams," Administrative Science Quarterly, vol. 44, no. 2, pp. 350-383, 1999.

[24] L. J. Gressgård and K. Hansen, "Knowledge exchange and learning from failures in distributed environments: The role of contractor relationship management and work characteristics," Reliability Engineering and System Safety, vol. 133, pp. 167-175, 2015.

[25] J. Liu, L. Geng, B. Xia, and A. Bridge, "Never let a good crisis go to waste: Exploring the effects of psychological distance of project failure on learning intention," Journal of Management in Engineering, vol. 33, no. 4, Article ID 04017006, 2017.

[26] I. Silla and N. Gamero, "Psychological safety climate and professional drivers' well-being: The mediating role of time pressure," Transportation Research Part F: Traffic Psychology and Behaviour, vol. 53, pp. 84-92, 2018.

[27] M. Baer and M. Frese, "Innovation is not enough: Climates for initiative and psychological safety, process innovations, and firm performance," Journal of Organizational Behavior, vol. 24, no. 1, pp. 45-68, 2003.

[28] A. Gegenfurtner, "Motivation and transfer in professional training: A meta-analysis of the moderating effects of knowledge type, instruction, and assessment conditions," Educational Research Review, vol. 6, no. 3, pp. 153-168, 2011.

[29] B. Schneider and A. E. Reichers, "On the etiology of climates," Personnel Psychology, vol. 36, no. 1, pp. 19-39, 1983.

[30] U. Safdar, Y. F. Badir, and B. Afsar, "Who can I ask? How psychological safety affects knowledge sourcing among new product development team members," The Journal of High Technology Management Research, vol. 28, no. 1, pp. 79-92, 2017.

[31] Y. Shen, M. M. Tuuli, B. Xia, T. Y. Koh, and S. Rowlinson, "Toward a model for forming psychological safety climate in construction project management," International Journal of Project Management, vol. 33, no. 1, pp. 223-235, 2015.

[32] R. Hirak, A. C. Peng, A. Carmeli, and J. M. Schaubroeck, "Linking leader inclusiveness to work unit performance: The importance of psychological safety and learning from failures," The Leadership Quarterly, vol. 23, no. 1, pp. 107-117, 2012.

[33] T. M. Probst and A. X. Estrada, "Accident under-reporting among employees: Testing the moderating influence of psychological safety climate and supervisor enforcement of safety practices," Accident Analysis \& Prevention, vol. 42, no. 5, pp. 1438-1444, 2010.

[34] A. Carmeli and J. H. Gittell, "High-quality relationships, psychological safety, and learning from failures in work organizations," Journal of Organizational Behavior, vol. 30, no. 6, pp. 709-729, 2009.

[35] S. Pemsel and A. Wiewiora, "Project management office a knowledge broker in project-based organisations," International Journal of Project Management, vol. 31, no. 1, pp. 31-42, 2013.

[36] A. Keegan and R. Turner, "Quantity versus quality in project based learning practices," Management Learning, vol. 32, no. 1, pp. 77-98, 2011.

[37] L. Lin and H. Wang, "Dynamic incentive model of knowledge sharing in construction project team based on differential game," Journal of the Operational Research Society, vol. 70, no. 12, pp. 2084-2096, 2019.

[38] L. Drupsteen and P. Hasle, "Why do organizations not learn from incidents? Bottlenecks, causes and conditions for a failure to effectively learn," Accident Analysis \& Prevention, vol. 72, pp. 351-358, 2014.

[39] E. H. Schein, Organizational Culture and Leadership, JosseyBass, SanFrancisco, CA, USA, third edition, 2004.

[40] B. Nguyen, J. Chen, and D. De Cremer, "When new product development fails in China: mediating effects of voice behaviour and learning from failure," Asia Pacific Business Review, vol. 23, no. 4, pp. 559-575, 2017.

[41] A. L. Tucker, I. M. Nembhard, and A. C. Edmondson, "Implementing new practices: An empirical study of 
organizational learning in hospital intensive care units," Management Science, vol. 53, no. 6, pp. 894-907, 2007.

[42] S. Liu, J. Hu, Y. Li, Z. Wang, and X. Lin, "Examining the crosslevel relationship between shared leadership and learning in teams: Evidence from China," The Leadership Quarterly, vol. 25, no. 2, pp. 282-295, 2014.

[43] E. S. Geller, "Are you a safety bully?" Professional Safety, vol. 59, no. 1, pp. 39-44, 2014.

[44] R. W. Thomas, T. L. Esper, and T. P. Stank, "Coping with time pressure in interfirm supply chain relationships," Industrial Marketing Management, vol. 40, no. 3, pp. 414-423, 2011.

[45] H. Li and Q. Zhang, "A literature review of learning from failure and prospects for future research," in Proceedings of the 2nd International Conference on Management Science and Innovative Education, pp. 487-493, Sanya, China, October 2016.

[46] A. Schmitt, S. Ohly, and N. Kleespies, "Time Pressure Promotes work Engagement," Journal of Personnel Psychology, vol. 14 , no. 1 , pp. $28-36,2015$.

[47] T. M. Amabile, "The social psychology of creativity: A componential conceptualization," Journal of Personality and Social Psychology, vol. 45, no. 2, pp. 357-376, 1983.

[48] J. Naveteur, S. Cœugnet, C. Charron, L. Dorn, and F. Anceaux, "Impatience and time pressure: Subjective reactions of drivers in situations forcing them to stop their car in the road," Transportation Research Part F: Traffic Psychology and Behaviour, vol. 18, pp. 58-71, 2013.

[49] A. L. Tucker and A. C. Edmondson, "Why hospitals don't Learn from failures: organizational and Psychological dynamics That inhibit system Change," California Management Review, vol. 45, no. 2, pp. 55-72, 2003.

[50] E. Kyndt, N. Govaerts, F. Dochy, and H. Baert, "The learning intention of low-qualified employees: A key for participation in lifelong learning and continuous training," Vocations and Learning, vol. 4, no. 3, pp. 211-229, 2011.

[51] W. Kim, H. M. Jun, M. Walker, and D. Drane, "Evaluating the perceived social impacts of hosting large-scale sport tourism events: scale development and validation," Tourism Management, vol. 48, pp. 21-32, 2015.

[52] J. F. Hair, R. Anderson, R. Tatham, and W. Black, Multivariate Data Analysis, Prentice Hall, Englewood Cliffs, NJ, USA, 5th ed. edition, 1998.

[53] R. M. Baron and D. A. Kenny, "The moderator-mediator variable distinction in social psychological research: Conceptual, strategic, and statistical considerations," Journal of Personality and Social Psychology, vol. 51, no. 6, pp. 1173-1182, 1986.

[54] W.-T. Wang and N.-Y. Ko, "Knowledge sharing practices of project teams when encountering changes in project scope: A contingency approach," Journal of Information Science, vol. 38, no. 5, pp. 423-441, 2012.

[55] D. D. Ahiaga-Dagbui, P. E. Love, S. D. Smith, and F. Ackermann, "Toward a systemic view to cost overrun causation in infrastructure projects: A review and implications for research," Project Management Journal, vol. 48, no. 2, pp. 88-98, 2016.

[56] S. McClory, M. Read, and A. Labib, "Conceptualising the lessons-learned process in project management: Towards a triple-loop learning framework," International Journal of Project Management, vol. 35, no. 7, pp. 1322-1335, 2017.

[57] X. Ferrada, D. Núñez, A. Neyem, A. Serpell, and M. Sepúlveda, "A cloud-based mobile system to manage lessons-learned in construction projects," Procedia Engineering, vol. 164, pp. 135-142, 2016.
[58] M. Terzieva and V. Morabito, "Learning from experience: The project team is the key," Business Systems Research Journal, vol. 7, no. 1, pp. 1-15, 2016.

[59] A. Khedhaouria, F. Montani, and R. Thurik, "Time pressure and team member creativity within R\&D projects: The role of learning orientation and knowledge sourcing," International Journal of Project Management, vol. 35, no. 6, pp. 942-954, 2017.

[60] T. O. A. Lehtinen, M. V. Mäntylä, J. Vanhanen, J. Itkonen, and C. Lassenius, "Perceived causes of software project failures An analysis of their relationships," Information and Software Technology, vol. 56, no. 6, pp. 623-643, 2014.

[61] Y. Shen, T. Y. Koh, S. Rowlinson, and A. J. Bridge, "Empirical investigation of factors contributing to the psychological safety climate on construction sites," Journal of Construction Engineering and Management, vol. 141, no. 11, p. 04015038, 2015.

[62] M. A. T. Lê and K. H. Law, "System dynamic Approach for simulation of Experience Transfer in the AEC industry," Journal of Management in Engineering, vol. 25, no. 4, pp. 195-203, 2009. 